\title{
Jams de Black Music en Buenos Aires: relaciones entre performances en vivo y sus mediatizaciones de Spotify
}

\author{
Emiliano Vargas \\ Facultad de Ciencias Sociales, Universidad de Buenos Aires. UBACYT. \\ emilianov1988@gmail.com \\ Fecha de finalización: 28 de agosto de 2020 \\ Recibido: 30 de agosto de 2020 \\ Aceptado: 1 de diciembre de 2020 \\ DOI: https://doi.org/10.26422/aucom.2020.0902.var
}

\section{Resumen:}

Este trabajo aborda las relaciones entre las instancias online y offline de las producciones musicales de Jams de Black Music (JBM), conjugando una perspectiva socio-semiótica de las mediatizaciones con una descripción del tipo de performance en vivo que construyen las JBM, las cuales se realizaron en el marco de doce observaciones participantes llevadas a cabo durante 2019; analizaron gracias a los aportes de distintos autores avocados al estudio de la improvisación musical y la performance artística afroamericana. Entre los resultados se destacan las características de las relaciones entre ambas instancias, el rol de la improvisación y los rasgos particulares de un tipo de performance en vivo no investigado hasta ahora, como así también las distintas transformaciones que introducen las mediatizaciones de Spotify y su proceso de uploading musical a las vidas de las JBM.

Palabras clave: black music, performance, improvisación, mediatización, Spotify.

\section{Black Music Jams in Buenos Aires: the relationship between live performances and Spotify mediatizations}

\section{Abstract}

In this paper, we analyze the musical productions that emerged from Black Music Jams in Buenos Aires and the relationship between their online and offline instances. We combine a socio-semiotic perspective on their mediatizations with a description of the kind of live performances carried out within the Jams, held in the context of twelve participant observations during 2019. We were able to analyze these performances thanks to the contributions of various authors who specialize in musical improvisation and in African American performance. Among our results, we highlight the relationship between offline and online instances, the role of improvisation, the characteristics of a sort of live performance that had not been investigated up until now, and the various transformations introduced by Spotify mediatizations, and their uploading process, into Black Music Jams.

Keywords: black music, performance, improvisation, mediatization, Spotify. 


\section{Jams de Black Music em Buenos Aires. Relação entre performances ao vivo e as suas mediações de Spotify}

\section{Resumo}

O trabalho trata das relações entre as instâncias online e offline das produções musicais de Jams de Black Music (JBM), combinando uma perspectiva sócio-semiótica das mediatizações com uma descrição do tipo de performace ao vivo que JBM constrói. Estas foram realizadas no âmbito das doze observações dos participantes realizadas durante 2019 e são analisadas graças às contribuições de diferentes autores dedicados ao estudo da improvisação musical e do espetáculo artístico afroamericano. Entre os resultados, destacam-se as características da relação entre ambas as instâncias, o papel da improvisação e as características particulares de um tipo de show ao vivo não estudado até agora, bem como as diferentes transformações introduzidas pelas mediatizações de Spotify e o seu processo de upload musical para a vida dos JBMs.

Palavras-chave: black music, performance, improvisação, mediatização, Spotify.

\section{Introducción}

El presente trabajo se desprende de una investigación mayor que analiza la construcción postbroadcasting de las Jams de Black Music (JBM) y se enfoca en las "vidas de lo musical" (Fernández, 2014; 2017), es decir, la vida social que transcurre en el sistema de producción, circulación y consumo de música offline-online y que involucra tanto a JBM como a músicos, públicos y usuarios de plataformas. Vidas que se van complejizando al calor de las transformaciones en sus ecosistemas mediáticos.

Se trata de un diseño flexible de investigación (Mendizábal, 2006, p. 68) en el que se elaboró una muestra diversa (Sampieri y Collaro, 2006, p. 567) con el fin de localizar diferencias y coincidencias, patrones y particularidades en las unidades de análisis. La muestra contiene dos JBM con distintas trayectorias: el caso A -de mayor trayectoria- y el caso B -de menor trayectoria-, las cuales transcurren en espacios culturales investigados por el proyecto de investigación en el que se inscribe el presente trabajo. ${ }^{2}$

\section{Las unidades de análisis y el sistema cultural}

La propuesta teórica se enmarca en el concepto de "cultura" de tradición lotmaniana (Lotman, 1998a, 1998b). Desde allí, la instancia online es abordada con una perspectiva socio-semiótica de los medios, teniendo en cuenta los sistemas de intercambios discursivos mediáticos a partir de los tres niveles que componen la mediatización

\footnotetext{
En el marco del proyecto de investigación en el que se inscribe el presente trabajo, se denomina postbroadcasting al momento mediático actual caracterizado, entre otras cosas, en la convivencia registrada entre los intercambios en broadcasting (de uno a muchos) y en networking (de muchos a muchos), o bien las nuevas relaciones que emergen entre fenómenos discursivos artísticos y sus mediatizaciones.

2 Proyecto de investigación con financiamiento UBACyT "Letra, imagen, sonido. Plataformas y redes sociales: relaciones entre mediatización, espacio urbano y cultura", Facultad de Ciencias Sociales, Universidad de Buenos Aires.
} 
musical $^{3}$ (Fernández, 2017, p. 92). Su instancia offline es reconstruida a partir de doce observaciones participante (OP) (Jociles Rubio; 2018; Kawulich, 2005), es decir, seis OP realizadas a cada unidad de análisis, a través de las cuales se describe el tipo de performance en vivo que construyen las JBM y que se analizaron gracias al aporte de autores avocados al estudio de la tríada improvisación musical-performance-composición proveniente de la musicología, la etnomusicología, la semiótica (Benson, 2003; López Cano, 2018; Nettl y Russel, 2004) y de los estudios de la performance artística afroamericana (Frigerio, 1992).

Se denomina Jams de Black Music (JBM) a un tipo de colectivos culturales que se encuentran en expansión en Buenos Aires. Con distintos niveles de trayectorias, convocatoria y organización realizan periódicamente performances en vivo con características no investigadas hasta ahora, a la vez que mantienen una activa vida online a través de sus ecosistemas mediáticos. Dentro de sus ecosistemas, el presente trabajo se ocupa de las mediatizaciones en Spotify.

Las JBM forman parte de la semiosfera de la Black Music (Lotman, 1998b, pp. 168213), una macroclass -geográfica/locativa/étnica- de géneros musicales (Marino, 2020, p. 102) que anida en su interior a microsistemas textuales lo suficientemente unidos, pero lo suficientemente separados, a través de fronteras, es decir, barreras con porosidades que permiten intercambios retóricos, temáticos $y /$ o enunciativos entre unos y otros. Los microsistemas textuales son los distintos géneros que, dentro de la macroclass, van adoptando diferentes grados de cristalización o liquidez con base en su longevidad, produciendo sentido no solo en materialidades sonoras, sino también visuales a través de operaciones de transposición (Greimas, 1968; Steimberg, 1993).

Mientras el blues y el jazz constituyen el núcleo de la semiosfera, a su alrededor se configuran otros microsistemas, como el funk, el neo-soul, el disco, el hip hop y el trap, acercándose a los márgenes de la semiosfera con diferentes grados de cristalización y liquidez. ${ }^{4}$

Autores avocados al estudio de la black music desde la sociología (Cole, 2013; Neal, 1999), la musicología (Ripani, 2006, pp. 3-9) o desde los llamados popular music studies (Tagg, 1989, pp. 1-18) no logran consenso en torno a una denominación común del fenómeno. Black music, blue music, afroamerican music o rhythm \& blues son solo algunos de los nombres que se propusieron desde el campo académico y la industria musical.

\footnotetext{
3 La mediatización se compone a partir de las relaciones entre sus tres niveles, los cuales se encuentran inmersos tanto en la descripción del presente artículo como también en trabajos anteriores (Vargas, 2020). Los tres niveles son: dispositivos técnicos, géneros y estilos musicales involucrados y usos y prácticas sociales (Fernández, 2017 p. 92 ).

4 Para acceder a una descripción de los géneros musicales más representativos de las JBM y sus características mediáticas, se recomienda la lectura del artículo de Vargas (2020).
} 
Emiliano Vargas

Jams de Black Music en Buenos Aires: relaciones entre performances en vivo y sus mediatizaciones de Spotify

Se ha optado por denominar a la semiosfera como black music en base a distintos criterios. En primer lugar, tomando las definiciones elaboradas por Mark Anthony Neal (1999) y Gabriele Marino (2020), en las que el primero aborda el fenómeno de modo holístico, atendiendo a su contexto histórico, musical y socio-étnico, mientras que Marino lo hace desde una perspectiva socio-semiótica que atiende a las complejidades que atraviesan los procesos sociales de clasificación de los géneros musicales.

Otros autores nombrados basan sus clasificaciones en criterios específicamente musicales (Ripani, 2006), hacen alusión al fenómeno con diferentes nombres en un mismo trabajo (Cole, 2013) o, en el caso de Philip Tagg (1989), el autor realiza una crítica de relevancia semiótica a los criterios de categorización con los que la musicología ha lidiado históricamente para arribar a denominaciones como black music o afroamerican music. Es decir, clasificaciones con cargas étnicas que, según Tagg (1989), refuerzan posiciones de dominación sobre las comunidades afroamericanas. Sin embargo, su crítica no incluye una propuesta que supere las contradicciones que señala.

El otro criterio reside en coincidir con el modo en el que las JBM denominan a la semiosfera (ver Figura 1).

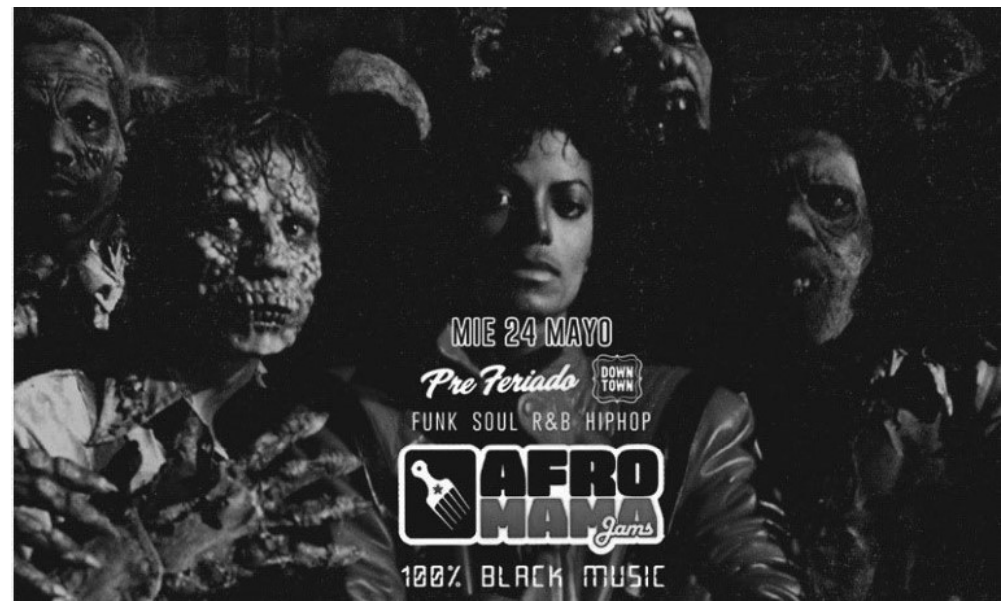

Figura 1. Promoción de un evento Afromama.

Los microsistemas de la semiosfera comparten elementos retóricos rastreables en la tradición de la música afronorteamericana visibilizada desde finales del siglo XIX en los Estados Unidos y expandida globalmente durante el siglo XX gracias al desarrollo de los medios de comunicación. Es decir, forman parte del devenir de la diáspora cultural africana que opera de distintos modos en el continente americano. Si bien los rasgos particulares que adopta la diáspora en Argentina se encuentran fuera de los objetivos del presente trabajo, se considera necesario subrayar algunos aspectos que serán desarrollados con mayor profundidad en futuras publicaciones. 
Austral Comunicación

Volumen 9, número 2 (Diciembre de 2020): 299-323. ISSN 2313-9129

\section{Sobre la cultura afro en Argentina}

En relación con la diáspora africana en Argentina, si bien los procesos migratorios africanos datan de principios del siglo XVIII en el marco de la trata de esclavos, no es menos cierto que su descendencia atravesó un proceso de desarrollo que tuvo como aliado al factor invisibilización en los siglos siguientes (Corti, 2015; Frigerio y Lamborghini, 2011).

En ese marco, la comunidad afro adoptó diferentes formas de comunalización, entre las que se destacan, en un primer momento, las cofradías de carácter exclusivamente religioso. En un segundo momento, las formas de comunalización pasaron a estar organizadas con base en las nacionalidades y sus posteriores formas de institucionalización, las cuales fueron mutando a lo largo de los siglos XIX y XX, adoptando rasgos similares a las sociedades europeas de Argentina -es decir, instituciones civiles formadas en Argentina a partir de las diversas comunidades de inmigrantes europeos- $y$ durante el siglo XXI a través de diversas formas organizativas (Frigerio y Lamborghini, 2011).

Alejandro Frigerio (2011) describe una creciente producción cultural afro y el incremento en la participación y adhesión a los eventos y prácticas por parte de la comunidad blanca y el conjunto de la sociedad no africana, así como también un aumento de la cantidad de eventos con fines exclusivamente recreativos durante los últimos veinte años.

En este sentido, se subraya que las expresiones musicales y bailables afroamericanas vinculables a la diáspora africana del Cono Sur -como, por ejemplo, el candombe argentino, el uruguayo o el pericón- no fueron registradas en las observaciones participantes realizadas a las unidades de análisis. Por ese motivo, y teniendo en cuenta las diferencias entre las distintas colectividades afro dentro de Argentina, se considera al conjunto de géneros musicales contenidos en la semiosfera como música de raíz afronorteamericana en tanto el núcleo del modelo remite al blues y al jazz (Vargas, 2020).

Se trata de un sistema cultural que evoluciona a partir de ambos géneros; en este sentido, es necesario mencionar una de las ideas centrales a las que arriba Berenice Corti (2015) en su libro Jazz Argentino. La música "negra" del país "blanco" al afirmar que la dificultad para hablar de un jazz argentino "reside en que, tanto local como internacionalmente, el jazz ha sido racializado como música negra" (p. 152). Para la autora, se trata de un aspecto fundamental en tanto impidió que el género estuviera asociado a los discursos de construcción de las músicas nacionales que cumplieron roles específicos de afirmación de las identidades locales en el marco de los proyectos latinoamericanos de Estado-nación en el siglo XX.

En el mismo trabajo, la autora se refiere a los vínculos entre música e identidad, afirmando que la identidad no sería una cosa o contenido ni un lugar al que arribar, 
Emiliano Vargas

Jams de Black Music en Buenos Aires: relaciones entre performances en vivo y sus mediatizaciones de Spotify

sino un tipo específico de significación o producto discursivo, de primero o segundo grado, ${ }^{5}$ con características propias y situadas. A la inversa, en tanto fenómeno social, la práctica musical es, al menos en una de sus dimensiones constitutivas, un proceso de producción de sentido(s) -por ejemplo, de identidad- (Corti, 2015, p. 27).

Es decir que, desde su punto de vista, la imposibilidad de construir un modo nacional de producción de música a partir de aquellos géneros previamente racializados no impide la gestación de construcciones identitarias o de diferentes procesos de identificación a partir de sus prácticas.

\section{Precisiones sobre las unidades de análisis}

Durante 2019 se realizaron seis OP a cada unidad de análisis con el objetivo de recopilar datos que permitan reconstruir y analizar el tipo de performance que construyen las JBM, atendiendo a sus coincidencias y diferencias. Las OP realizadas a Afromama fueron llevadas a cabo en calidad de público asistente, mientras que en el caso de Skill Session -gracias a un mayor grado de apertura a la participación de diferentes músicos dentro de su propuesta- la participación se llevó a cabo también en calidad de músico sobre el escenario en tres oportunidades.

Las unidades de análisis seleccionadas resultan de interés en tanto transcurren en espacios culturales, los cuales aborda el proyecto de investigación en el que se inscribe el presente trabajo, y, además, porque se encuentran en estadios opuestos de desarrollo, aspecto que enriquece el análisis de relaciones. Afromama (caso A) posee un trayectoria considerable en el circuito de espacios culturales de Buenos Aires, y Skill Session (caso B) es una JBM nueva, de menor trayectoria, que da los primeros pasos en el desarrollo de su propuesta.

El caso A existe desde hace 16 años, es decir, nació dentro del período de proliferación de la cultura afro en Buenos Aires y, desde entonces, lleva a cabo sus performances todos los domingos en un espacio llamado Makena Cantina Bar -situado en el barrio porteño de Palermo- ${ }^{6}$ aunque en ocasiones las realiza de modo itinerante por distintos puntos de Buenos Aires y el país. 7 Posee un alto nivel de organización y convocatoria en el que aglutina a alrededor de mil personas cada domingo.

\footnotetext{
Corti se refiere a los discursos musicales de primer grado, siguiendo a José Jorge Carvalho y Rita Segato (1994), denominándolos como musicopoiesis. Es decir, el discurso propiamente musical que emerge en el acto de hacer música. Por su lado, los de segundo grado tienen que ver con los que producen los nativos al referirse a la música, es decir, corresponde a un plano metadiscursivo.

6 Un registro periodístico de su trayectoria y algunas de sus características puede leerse en: Afromama, música negra en el... (2018). En relación con los registros bibliográficos en su libro Nunca es demasiado (2019), el célebre baterista argentino Fernando Samalea se refiere a Afromama como un espacio de referencia dentro del circuito de black music, el cual solía frecuentar junto a distintos músicos y amigos.

7 Un registro periodístico de una de las celebraciones de Afromama en la ciudad de Rosario puede leerse en: Vuelve la fiesta Afromama (2017).
} 
Austral Comunicación

Volumen 9, número 2 (Diciembre de 2020): 299-323. ISSN 2313-9129

Sus performances en vivo son ejecutadas por un staff de músicos relativamente estable, denominado Afromama Crew, aunque, en ocasiones, se invita al escenario a artistas consagrados de la black music, entre los que se destacan Dante Spinetta y Ca7riel (ver Figura 2), los cuales fueron registrados durante las OP.

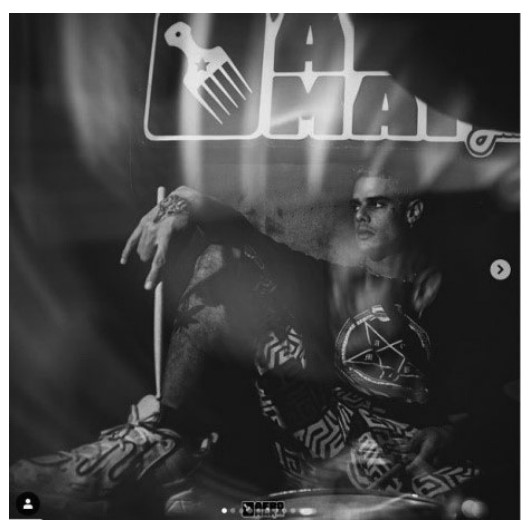

Figura 2. El artista C7riel, invitado a una performance en Afromama.

El caso B es una JBM con cinco años de vida. Sus eventos en vivo se realizan los terceros viernes de cada mes de modo itinerante en distintos espacios culturales de la Ciudad de Buenos Aires y del conurbano bonaerense, como La Gran Jaime, Casa Reacción o El Emergente. La participación de los músicos es abierta, aunque existe un grupo de artistas que participa con mayor asiduidad (ver Figuras 3 y 4 ). A diferencia del caso A, el único registro bibliográfico existente sobre Skill Session es un artículo publicado en el que se analizan las mediatizaciones de ambos colectivos en la plataforma Instagram (Vargas, 2020).

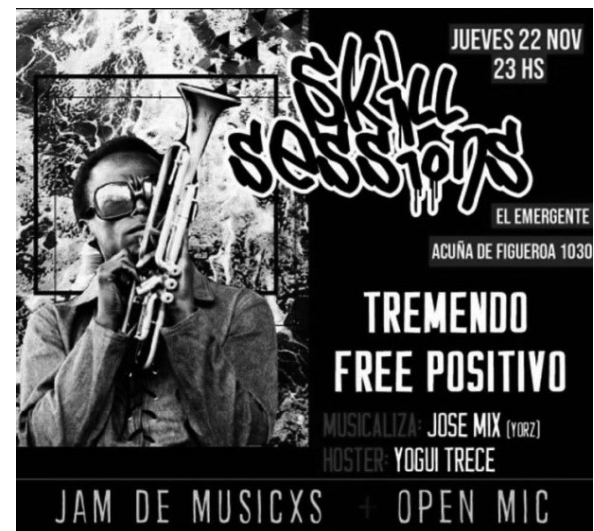

Figura 3. Flyer promocional de Skill Session. 
Emiliano Vargas

Jams de Black Music en Buenos Aires: relaciones entre performances en vivo y sus mediatizaciones de Spotify

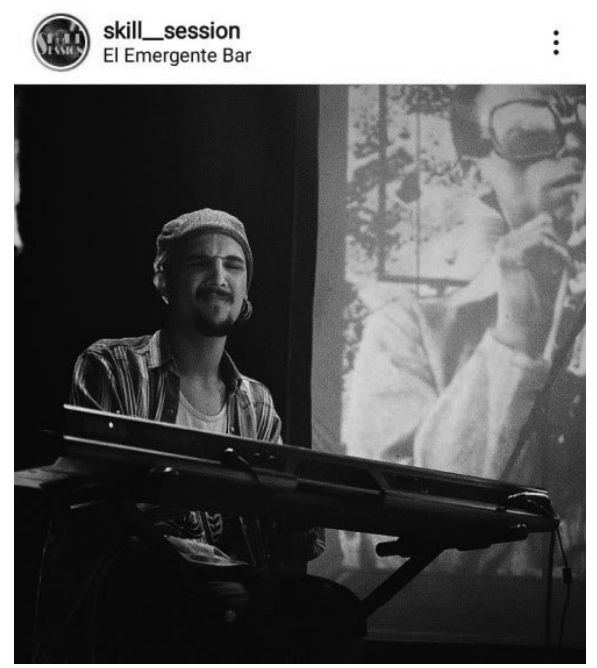

Figura 4. Músico en plena performance en Skill Session.

\section{Performance en vivo - performance grabada}

Resulta relevante destacar el aporte de algunos investigadores provenientes de la musicología, quienes se han encargado de subrayar la complejidad de los vínculos entre performances en directo y performances grabadas. Si bien referirse a instancias online y offline no necesariamente es lo mismo, ya que ambas pueden contener perfomances en directo - por ejemplo, transmisiones de un concierto en vivo vía Instagram, Facebook o YouTube-, se subraya que las mediatizaciones de Spotify analizadas en el presente trabajo están basadas en el registro y la conservación técnica de fonogramas.

Rubén López Cano (2018) divide distintas etapas en la historia de la música occidental y denomina al momento actual como reino de la música grabada. En ese marco, el autor afirma que si bien "la interpretación en directo es fenomenológicamente distinta, en esta etapa la grabación pasó a propiciar una forma particular de interpretación musical en sí misma” (pp. 163-180).

Es decir, la performance grabada no necesariamente comparte la misma ontología que la interpretación en directo, pero ambas se codeterminan. Como menciona Auslander, "se trata de valoraciones construidas históricamente. De hecho el concepto de música en vivo emerge y tiene sentido solo después que existe la música grabada" (Auslander, 2008, como se citó en López Cano, 2018, p. 179).

Por otro lado, Nicholas Cook (2013) plantea que "escuchar una grabación es experimentar la música como una performance, y de esta forma las performances grabadas o en vivo, en conjunto, constituyen un único, aunque variado y complejo dominio cultural. Visto de esta manera, los productores e ingenieros de sonido se convierten en intérpretes" (p. 6). 
Austral Comunicación

Volumen 9, número 2 (Diciembre de 2020): 299-323. ISSN 2313-9129

Rubén López Cano (2018) también afirma que, a mediados del siglo XX, los patrimonios musicales de géneros contenidos en la semiosfera (el autor nombra explícitamente a la música disco y al hip hop)

Estaban pasando por un proceso intenso de objetualización ya sea a través de registros estables como grabaciones fonográficas o por medios conceptuales y discursivos (historia de la música, el análisis musical como institución didáctica, el canon expresado en libros, programas de concierto y crítica, etc.). Parece ser que en algún punto, los y las compositoras, creadoras, productoras y postproductoras coincidieron en el deseo de que obras reconocibles e identificables abandonaran su condición de Archivo que preserva la memoria del pasado, para convertirse en Repertorio o materia prima orgánica para acceder al futuro, para seguir produciendo cultura" (p. 135).

Para el musicólogo, archivo y repertorio implican operaciones culturales diferentes. Mientras que el archivo encapsula y custodia la música como patrimonio preservando sus discursos valorativos y aproximándose a ellos desde una perspectiva histórica, el repertorio trabaja orgánicamente con el patrimonio para seguir produciendo cultura, es decir, para hacer algo a través de él.

Por lo tanto, no solo es posible hablar de performance grabada y performance en vivo, sino que, además, ambas categorías parecen contenerse como una totalidad. Pero, tal como afirmó Cook (2013), el tipo de relaciones que se pueden establecer entre ambas instancias es complejo y variado. El objetivo, entonces, es reconocer y describir esa complejidad.

\section{Análisis del tipo de performance offline}

Las performances en vivo de las JBM son denominadas jams dentro de la jerga. A su vez, este término se vincula semánticamente con la acción de improvisación musical.

Desde un punto de vista etimológico, la palabra improvisación proviene del término latino improvisus, que significa imprevisto.

Dentro de la música popular occidental, las sesiones de improvisación comenzaron a ser nombradas como jam sessions en el ámbito del jazz, género que, a su vez, coincide con una parte del núcleo de la semiosfera. La jam session pone énfasis en la idea de improvisación como práctica asociada a la composición y/o performance extempore, es decir, sin preparación previa (Latham, 2017, p. 757).

El Diccionario enciclopédico de la música (Latham, 2017) define a las jams sessions como reuniones de jazzistas en la que los intérpretes improvisan de manera informal y sin un plan preconcebido. En la década del 30, comenzó a asociarse con sesiones de improvisación en las que músicos que trabajaban en las big-bands podían disfrutar de una mayor libertad creativa al momento de estos encuentros (p. 801). 
Emiliano Vargas

Jams de Black Music en Buenos Aires: relaciones entre performances en vivo y sus mediatizaciones de Spotify

El estudio de la improvisación musical (IM) es relativamente nuevo tanto en la musicología como en la etnomusicología y la semiótica. Tanto Eero Tarasti (2002) como Bruno Nettl y Melinda Russell (1998) destacan a Ernst T. Ferand como el único investigador que abordó el fenómeno durante décadas, "proporcionando una profundidad histórica etnográfica y sistemática, y demostrando que [la improvisación musical] tenía diversos significados para diversos pueblos, épocas y grupos de estudiosos" (Nettl y Russell, 1998, p. 9).

Distintos investigadores coinciden en que un análisis de la IM debe aceptar la relación indisoluble de la tríada composición-performance-improvisación (Benson, 2003; Nettl y Russell, 1998).

$\mathrm{Al}$ respecto, Bruce Ellis Benson (2003) señala que la performance de una pieza musical puede transcurrir entre dos ideales o polos que denomina, citando a William Desmond, encapsulación -aludiendo a la acción de reproducir música tal cual es en su versión original-y participación -en la que se libran modificaciones de distinta índole durante el momento de la ejecución, sean composiciones de arreglos o improvisaciones- (Desmond, 1986, como se citó en Benson, 2003, p. 22).

Benson (2003) expone once tipologías de improvisación musical que, en el marco de esta investigación, fueron reelaboradas en seis categorías que coexisten dentro del tipo de performance que construyen las JBM. Su reconocimiento permite una descripción más detallada de los recursos utilizados para las variaciones que se dan entre los ideales de encapsulación y participación.

La reelaboración de las categorías fueron aplicadas a los distintos recursos musicales registrados durante las OP, manteniendo solo aquellas que pueden ser utilizadas por fuera de la música culta occidental (Nettl y Russell, 2004, p. 16), es decir, aquellas que, según Benson (2003), "se encuentran tanto en la música barroca como en el jazz" (pp. 28-31) y su ejecución -al igual que las unidades de análisis- no depende, o depende parcialmente, del uso de la partitura.

Improvisación A: la línea melódica original es modificada ligeramente, de modo que la pieza musical es claramente reconocible.

Se trata de la categoría más utilizada en el tipo de performance analizado, en tanto que la ejecución de versiones (López Cano, 2018) es el recurso más común en ambos casos, aunque no se realizan bajo el ideal de encapsulación, sino que se acercan en diferentes grados al ideal de participación mediante el uso de arreglos e improvisaciones, las cuales no obturan el reconocimiento de la pieza original por parte del público.

Improvisación B: es igual que la A, pero a nivel armónico. Los acordes son alterados lo suficiente como para que la obra adquiera sonoridades distintas a la original, pero, aun así, se mantienen lo suficientemente cerca de la versión de referencia para que el nuevo acorde "encaje" en el lugar del acorde original. 
Unos de los recursos más usados en este tipo de alteraciones es la sustitución de acordes con base en los grados de una escala diatónica cuando se trata de música inscripta en el sistema tonal. ${ }^{8}$ Por ejemplo, en la música popular occidental, la sustitución del I grado de la escala mayor por el III (menor) de la misma escala o bien por el VI (menor). También es común la sustitución del II grado por el V o el VI. Mientras que en el primer caso la sustitución se realiza dotando a la armonía de un clima que genera un efecto de tranquilidad o reposo, en el segundo caso busca generar un efecto de tensión.

Improvisación C: la línea melódica es modificada sustancialmente acercándose al ideal de participación. La conexión con la melodía original ya no es completamente discernible para el oyente.

Se trata de un recurso que utilizan las JBM cuando la performance es desarrollada dentro del jazz, aunque también se puede aplicar al funk y al neo-soul, que -dentro del espectro de la black music- comparten recursos armónicos y melódicos del primer género mencionado. Por ejemplo, cuando se trata del solo de un instrumento, generalmente de guitarras, teclados, bajos e instrumentos de viento. Un recurso frecuente es la utilización de escalas modales ${ }^{9}$ sobre solos de instrumentos que, en su versión original, se estructuran sobre escalas diatónicas.

Improvisación D: ciertos acordes son cambiados sustancialmente, aunque la estructura básica de la pieza permanece intacta. Se trata de un recurso que puede llevarse a cabo de modo similar a la categoría $\mathrm{B}$, aunque con mayor densidad, por medio de sustituciones armónicas que modifican la percepción de las melodías que suenan sobre ella.

Improvisación E: la melodía es completamente ignorada, siendo reemplazada por una melodía alternativa -o, simplemente, ninguna melodía discernible- en su lugar.

Improvisación F: utiliza la partitura como guía -por ejemplo, una típica pieza de blues de dieciséis compases- y el intérprete improvisa dentro de esos confines. Puede que no haya ninguna conexión con la melodía original o incluso con los acordes. Mientras que las categorías anteriores tienen alguna conexión con la versión original, aquíla conexión puede no existir o ser casi nula, al menos para el oyente. Es la tipología

\footnotetext{
El sistema tonal utiliza dos sucesiones diferenciadas de sonidos: el modo o escala diatónica mayor y el modo o escala diatónica menor. Cada una de las transposiciones de los modos constituye una tonalidad, reuniendo un total de veinticuatro tonalidades que, a su vez, poseen siete grados distintos con base en los intervalos de las escalas diatónicas.

9 La historia de la música occidental reconoce al sistema modal como proveniente de la Edad Media y del Renacimiento. El sistema modal utiliza diferentes sucesiones de sonidos, los modos: jónico, dórico, frigio, lidio, mixolidio, eólico, locrio, siendo en total siete sucesiones que se pueden construir desde cualquier nota. Cada modo comienza en una nota puntual y tiene una estructura característica asociada a un carácter expresivo o emotivo desde el que se construye la intención modal.
} 
Emiliano Vargas

Jams de Black Music en Buenos Aires: relaciones entre performances en vivo y sus mediatizaciones de Spotify

menos frecuente en las JBM y, generalmente, aparece cuando se interpretan estándares pertenecientes al núcleo de la semiosfera.

En síntesis, las tipologías registradas con más frecuencia fueron las A, B, C, D y E y, con menor frecuencia, la F. Se ejecutan distintos tipos de versiones que, mediante el uso de las tipologías, se mueven entre los ideales de encapsulación y participación. Es decir, la improvisación entra en juego como un elemento más para lograr los movimientos entre los ideales mencionados.

Los diferentes grados de variación existentes entre el ideal de encapsulación y participación pueden ser descriptos con mayor precisión gracias al aporte de Rubén López Cano (2018, pp. 208-210) en sus estudios sobre la versión en la música popular. El autor distingue entre las nociones de: (i) réplica o cover; (ii) covering-interpretación leve e interpretación mayor-; y (iii) canción paralela.

La réplica o cover pretende ser lo más parecido posible a la versión de base o referencia, reproduciendo timbre, fraseo y sonido general de las grabaciones de referencia.

En el covering, la pieza es transformada en mayor o menor medida para ser adaptada al estilo del cantante o banda que realiza la versión de referencia. Esta categoría, a su vez, se divide en interpretación leve, la cual busca mantener determinados aspectos de la versión de referencia -como, por ejemplo, melodías, letras, instrumentación, a la vez que varía en algunos de sus elementos en la performance de sus intérpretes- y la interpretación mayor, en la que las transformaciones adquieren mayor intensidad. El autor destaca entre las transformaciones los cambios de estilo o género de la versión de base, los cuales son muy frecuentes en el tipo de performance construido por las JBM, por ejemplo, a través de la ejecución de versiones funk de piezas que originalmente pertenecen al pop o al jazz.

Finalmente, en la canción paralela, la versión dista aún más de la pieza original, en la que si bien es posible encontrar los elementos característicos de la versión de referencia, estos aparecen descontextualizados y/o fragmentados.

Es decir, se trata de diferentes grados de fluctuaciones entre los ideales mencionados con respecto a la versión de referencia, siendo la réplica o cover y ambas variedades de covering los tipos de versión más frecuentes en las JBM, mientras que la canción paralela ha sido registrada en ocasiones puntuales y de menor frecuencia.

A los tipos de versión descriptas se suma el registro esporádico del freestyle, ${ }^{10}$ aunque no en la modalidad de batallas (Vega, 2020, p. 67), sino como un recurso que aparece fusionado en la interpretación mayor dentro del covering.

También se destaca la figura del disc jockey (DJ), quien, en el caso de Afromama, recupera rasgos provenientes de la música electrónica, como por ejemplo la disposi-

10 Se denomina freestyle al rap creado en el momento. Es decir, una improvisación ex tempore de índole verbal en la que el freestyler expresa lo que ve o lo que siente sin perder el ritmo de la música o beat que suena de fondo. 
ción de la cabina ${ }^{11}$ en un lugar de relevancia dentro de la distribución del espacio físico donde se desarrolla el evento y desde el que se pincha ${ }^{12}$ música, a partir de la combinación de vinilos con bandejas digitales mediante la utilización del software VST, ${ }^{13}$ mezclando así herramientas y sonoridades analógicas y digitales.

En el caso A, el rol del DJ coincide con la descripción del disc jockey realizada por Víctor Lenarduzzi (2012, pp. 79-80), en tanto su figura ocupa un lugar destacado en el que interactúa con el público no solo a través del feedback generado con la música, sino también a través del movimiento de su propio cuerpo.

A partir de la figura del DJ se han registrado en el tipo de perfomance tanto mashups como remix (López Cano, 2018, pp. 284-286).

Mientras la primera definición hace referencia a una pieza musical formada por extractos de pistas previas, la segunda se trata de una remezcla o reciclaje digital realizado a partir del uso de vinilos o el uso de softwares como VST.

En el caso de Skill Session, la figura del DJ se diluye, omitiendo la utilización de bandejas de vinilos y relegando la musicalización a los organizadores y músicos a partir de los reproductores de música de ordenadores y smartphones.

Se destaca que, en ambos casos, el reconocimiento de la pieza original por parte del público es parte constitutiva del tipo de performance que construyen las JBM, desde la que se gesta un vínculo comunicacional a través del canto de las piezas musicales y el baile.

En este sentido, se señala que la definición de jam session provista por el Diccionario enciclopédico de la música (Latham,2017) no coincide con el tipo de performance construido por las JBM, lo cual denota una expansión semántica del concepto en los colectivos musicales analizados en relación con las sesiones de improvisación de principios del siglo XX.

La imprevisibilidad de la performance pierde terreno ante el cover, el covering y la canción paralela, ponderando la necesidad de reconocimiento de la pieza ejecutada y la introducción de otros elementos, como el freestyle o la figura del DJ.

\section{Análisis de la instancia online}

El análisis de mediatizaciones en Spotify ${ }^{14}$ permitió discernir dos aspectos que se consideran relevantes. Por un lado, el uso de playlists en ambas JBM y, por otro, el pro-

\footnotetext{
${ }^{11}$ Con respecto al rol y a las características del DJ en la música electrónica y sus nexos históricos con la black music, se recomienda la lectura de Placeres en Movimiento. Cuerpo Música y Baile en la Escena Electrónica (Lenarduzzi, 2012).

12 La acción de pinchar música hace referencia dentro de la jerga a la práctica del DJ de reproducir y mezclar fonogramas durante una performance.

13 Virtual Studio Technology (VST) es una interfaz estándar desarrollada para conectar y emular sintetizadores de audio para su edición, secuenciación y grabación.

${ }^{14}$ Spotify fue lanzado oficialmente el 7 de octubre de 2008, luego de acordar licencias con las grandes compañías discográficas tras dos años en versión de prueba. Junto a la apertura de los servicios de pago, las cuentas gratuitas limitadas por invitación fueron extendidas gradualmente, llegando en 2009 al Reino Unido. En la actualidad, la plataforma cuenta con 50 millones de piezas sonoras en su plataforma de streaming.
} 
Emiliano Vargas

Jams de Black Music en Buenos Aires: relaciones entre performances en vivo y sus mediatizaciones de Spotify

ceso de uploading musical en Spotify, lo que implicó el registro de novedades -en el caso A- que se consideran importantes.

\section{Playlists}

Las playlists son listas de reproducción de fonogramas seleccionados por el usuario, con las que otros usuarios pueden interactuar de diversos modos. ${ }^{15}$ Ambas JBM han confeccionado playlists que poseen rasgos individuales.

La playlist de Afromama está compuesta por clásicos de black music, entre los que se destacan Parliament Funkadelic, Michael Jackson, Prince, James Brown o The Sly and the Family Stone, Stevie Wonder, Marvin Gaye y Bootsy Collins, combinados con piezas musicales que varían entre el hip hop y el neo-soul y sonoridades que a veces toman elementos del pop, entre los que se destacan D'Angelo, Thundercat, 2Pac, Anderson Paak, TLC, Kendrick Lamar, Snoop Dog y Erykah Badu. En menor medida, y hacia el final de la lista, se registró música nacional, puntualmente Illya Kuryaki \& The Valderramas, Emme, NAFTA, Wos, Cat7riel y Duki.

Se destaca que, entre los primeros diez fonogramas, se registraron intercaladas piezas musicales editadas por Afromama en su perfil de artista verificado, el cual se describe en el siguiente apartado.

La playlist de Skill Session registró una mayor presencia de artistas locales. Si bien se encontraron artistas internacionales, la cantidad es menor en relación con la playlist de Afromama. Los principales rasgos que la diferencian de esta son la ausencia de clásicos de black music, como también de artistas consagrados. Ya sean internacionales o nacionales, los músicos que la componen poseen un menor nivel de reconocimiento.

Este aspecto deja espacio a un componente futurista más amplio, audible a partir de una mayor presencia de sintetizadores digitales, un uso reiterado del efecto denominado vococher-logrado a través del auto-tune $e^{16}$ en las voces- y percusiones construidas con cajas de ritmos digitales, las cuales caracterizan el sonido de la playlist. Su uso constituye, además, un efecto de época de la semiosfera.

Si bien estos rasgos también fueron registrados en la playlist de Afromama al reproducir a artistas como Thundercat, Anderson Paak, Ca7riel o Duki, el perfil sonoro que construye contiene un abanico más amplio y representativo de la semiosfera, en el

\footnotetext{
15 Además de su reproducción, Spotify permite al usuario otros tipos de interacción, por ejemplo, las acciones de megustear, compartir el fonograma dentro y fuera de la plataforma o incluirlos en nuevas playlists elaboradas por el usuario o, incluso, por un conjunto de usuarios.

${ }_{16}$ Auto-tune es un procesador de audio utilizado tanto para corregir errores de afinación como para generar efectos en las voces. Se denomina vococher a un efecto utilizado en géneros como el trap, el hip hop, el pop y el neo-soul, aunque fue popularizado por el grupo Daft Punk en su disco Homework (1997).
} 
que se ha evidenciado un componente vintage más fuerte a partir de la presencia de los clásicos mencionados, que pertenecen principalmente al funk. ${ }^{17}$

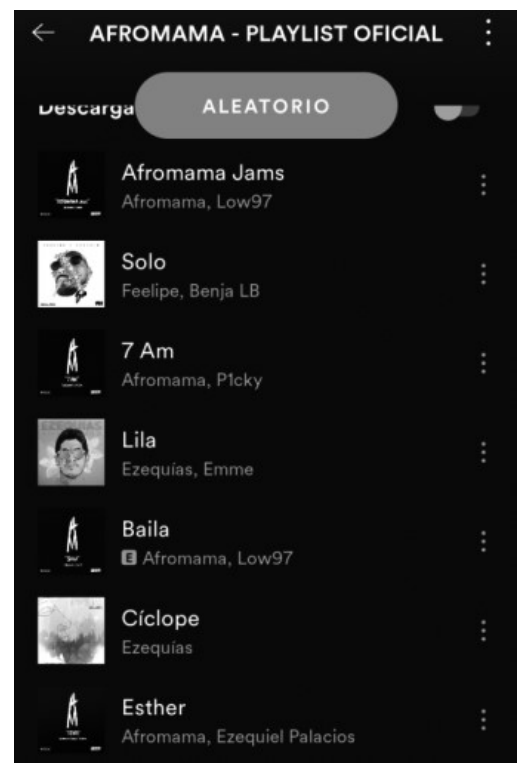

Figura 5. Fragmento de la playlist de Afromama.

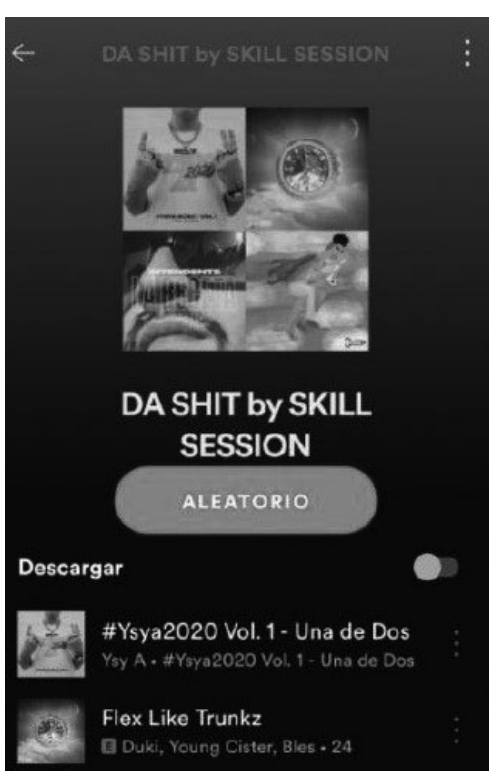

Figura 6. Fragmento de la playlist de Skill Session.

La playlist de Skill Session se caracteriza por contener fonogramas con un mayor nivel de fusión de géneros. Para distinguir los tipos de fusiones, se han elaborado dos polos distintivos, los cuales no son estáticos, sino que sirven como guía para una descripción más detallada.

Por un lado, el polo que fusiona hip hop y trap, con artistas como Linx, Intendente, Mattiya, Bizarrap, Cazzu, Sara Hebe, Micu Sierra y Emme.

Por otro lado, el polo neo-soul, funk, pop, con la presencia de Grace \& Bad Love, Sons of The James, Rob Milton, Palta \& The Mood.

En síntesis, la playlist de Afromama posee un estilo catálogo en tanto contiene una síntesis de géneros que han alcanzado determinado nivel de cristalización dentro de la semiosfera, combinados con fonogramas editados por la JBM cuyos autores se encuentran dentro de su staff de músicos.

Por su parte, la playlist de Skill Session evidenció un estilo emergente en tanto exhibe artistas de menor trayectoria, nacionales e internacionales, dentro del cual se

\footnotetext{
17 Si bien el Funk de la década del 60 y del 70 se ha caracterizado por exhibir la estética afrofuturista, sus huellas sonoras difieren de los rasgos futuristas que construyen actualmente músicos de hip hop o trap. Esta diferencia es audible, por ejemplo, a partir de un incremento en el uso de instrumentos digitales en detrimento de los analógicos. La elección no suele responder solo a criterios estéticos, sino también económicos que tienen como consecuencia un efecto de época en la semiosfera.
} 
destaca un predominio de músicos argentinos. Los fonogramas contienen fusiones de mayor liquidez entre géneros que se acercan a los márgenes de la semiosfera.

A través de las playlists, cada JBM elabora procesos de identificación que las diferencian de otras JBM, contribuyen a la circulación de fonogramas pertenecientes a artistas locales de bajo reconocimiento y contienen los ideales de encapsulación de las versiones de base que se ejecutan en la instancia offline.

\section{El proceso de uploading musical en Spotify}

Se destaca que diferentes autores, entre los que se puede nombrar a Jimena Jáuregui (2015), Benjamin Morgan (2020) y Jack Webster (2020), abordan el estudio de Spotify desde objetivos que depositan su interés en el análisis de diferentes usos y prácticas sociales vinculadas al usuario/oyente. El siguiente apartado indaga en la construcción de otro tipo de usuarios de Spotify que, sin embargo, se consideran relevantes. Se trata de los usuarios/creadores de contenido.

Spotify mantiene acuerdos con empresas discográficas, a la vez que ofrece la posibilidad a cualquier internauta de subir contenidos a sus plataformas a través del proceso de uploading musical.

La evolución histórica de las mediatizaciones implicó, durante el siglo XXI, el paso de la cultura downloading ${ }^{18}$ a la streaming, manteniendo como rasgo de permanencia la acción del uploading musical en los fenómenos postbradcasting (Fernández, 2017).

En algunas plataformas de streaming, como YouTube, el uploading se realiza de modo directo, es decir, usuario-plataforma con la intermediación opcional de otros actores. En el caso de Spotify, la empresa prevé -desde 2019- un proceso en el que es obligatoria la intervención de otras plataformas mediáticas por medio de un intercambio de servicios por sistema de pago ${ }^{19}$ a través de las llamadas distribuidoras digitales de contenidos,$^{20}$ con las que se realiza el uploading según los siguientes pasos:

\footnotetext{
${ }_{18}$ La cultura downloading se basa en la práctica del uploading de archivos a plataformas que permiten su posterior descarga para el consumo. El downloading tuvo su momento de auge entre la década del 90 y la primera parte del siglo XXI a partir de la irrupción de sitios como Napster, Ares o Torrent. Otra característica -en las mediatizaciones del sonido- es que puso en jaque a la estructura que configuraba hasta entonces a la industria musical (Fernández, 2014, 2017).

19 Se puede acceder a un artículo periodístico sobre el nuevo proceso de uploading en Spotify en: Spotify eliminará un servicio... (2019).

${ }^{20}$ Las distribuidoras o agregadoras digitales de contenidos son plataformas mediáticas que asumen un rol intermediario entre los usuarios y las plataformas de streaming musical. Entre algunas de las más reconocidas se encuentran Imusician (https://imusician.app/signin) y CdBaby (https://es.cdbaby.com/).
} 
Paso A: el usuario debe acceder al sitio de la distribuidora, crear un perfil y elegir entre los diferentes paquetes de servicios, en los que los precios varían según la cantidad de contenido que se sube y las plataformas streaming donde se requiere distribución. ${ }^{21}$

Paso B: seleccionado el paquete, el usuario sube los archivos a la plataforma de la distribuidora. Para cada producto - podcast, single, EP o álbum- se necesitan dos archivos: uno sonoro -en formato WAV, MP3 o WMA- y uno de imagen, generalmente JPG. Algunas distribuidoras, como Imusician, prevén en su interfaz la posibilidad de crear portadas para los productos mediante la utilización de plantillas gráficas.

Paso C: una vez subido el contenido, se agregan los metadatos ${ }^{22}$ del producto: nombres de artistas, nombre de la/las obra/s, datos de los compositores, arregladores e intérpretes, fecha de lanzamiento, idioma del título del lanzamiento, año de los derechos de autor, nombre del propietario de los derechos de autor, autor de la portada, año de derechos de autor de la portada y una clasificación de géneros y subgéneros a partir de categorías ofrecidas por la plataforma.

Distribuidoras como Imusician han comenzado a ofrecer un servicio de masterización instantánea ${ }^{23}$ es decir, además de ofrecer servicios de circulación, brindan la opción de finalizar la producción del fonograma de modo online, o sea, dentro de la plataforma.

Paso D: la agregadora distribuye los contenidos hacia las plataformas de streaming y notifica al usuario la fecha de lanzamiento del producto.

Spotify cuenta con dos plataformas que actúan de modo paralelo: Spotify (streaming) y Spotify for Artists. Mientras que la primera es una plataforma para usuarios/ oyentes, la segunda funciona para usuarios/creadores de contenido.

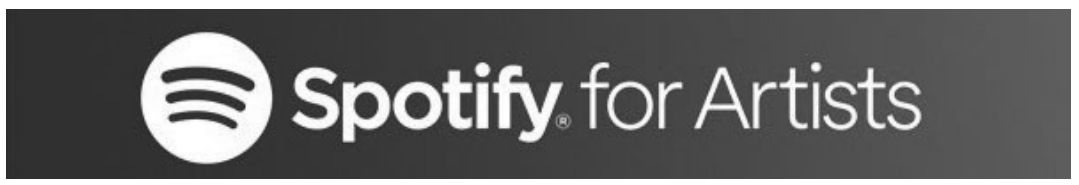

Figura 7. Isotipo de Spotify for Artists (Fuente: sitio oficial Spotify for Artists).

\footnotetext{
${ }^{21}$ Los paquetes de servicios no solo ofrecen distribución digital, sino también servicios de distribución de CD y vinilos a nivel mundial, obtención de licencias de sincronización para TV, películas y videojuegos, registro directo de canciones, afiliación a sociedades de gestión de derechos de ejecución en distintos países y derechos digitales de autoría, herramientas de marketing y recaudación de regalías.

22 Los metadatos pueden variar levemente según cada distribuidora; las descripciones responden a una síntesis resultante entre las plataformas Imusucian, CBBaby y Tunecore.

${ }^{23}$ La masterización es considerada el último paso en la producción de una pieza sonora. Su propósito es el de equilibrar los elementos sonoros de una mezcla -momento previo al mastering-y optimizar la calidad de su reproducción en todos los formatos y sistemas de reproducción.
} 
Emiliano Vargas

Jams de Black Music en Buenos Aires: relaciones entre performances en vivo y sus mediatizaciones de Spotify

Spotify for Artists realiza la recepción y gestión del contenido distribuido por las agregadoras, brindando acceso al usuario/creador de contenido a un sistema de tráfico de datos ${ }^{24}$ que se visualiza en formato gráfico, cuyo objetivo es proporcionar información orientada a maximizar la circulación de los fonogramas. La plataforma también propone un servicio de correo electrónico que funciona por defecto, en el que se provee información útil para mejorar la autogestión del músico en un contexto mediático que se transforma constantemente.

El perfil de artista -es decir, el perfil de usuario/creador de contenido- en Spotify for Artists es creado durante el proceso del primer uploading musical de modo paralelo al perfil de usuario creado en el sitio de la distribuidora. Los datos de usuario introducidos en ambos perfiles deben coincidir (nombre de usuario, nombre de artista y casilla de correo electrónico).

Paso E: cuando el contenido distribuido llega a Spotify for Artists, la plataforma envía un correo electrónico de verificación de identidad a la casilla del usuario. Una vez comprobada la identidad, la cuenta de artista verificado es activada con el primer contenido listo para ser reproducido por usuarios/oyentes desde la plataforma de streaming de Spotify (ver Figura 8).

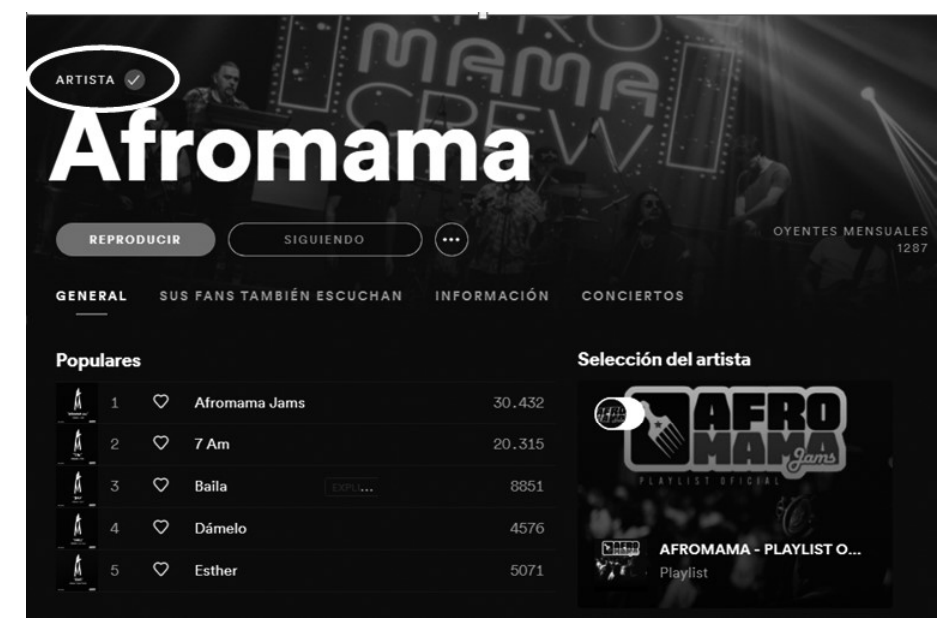

Figura 8. El contorno color blanco en la esquina superior izquierda muestra el logo de verificación del artista. (Fuente: perfil de Afromama en Spotify).

\footnotetext{
${ }_{24}$ El tráfico de datos que ofrece Spotify for Artists contiene variables de género (masculino, femenino, no especificado), fluctuaciones temporales de las reproducciones y edad de los oyentes. También incluye un ranking de países y ciudades con mayor cantidad de reproducciones y las fuentes de acceso desde donde se reprodujo el contenido.
} 
El perfil de artista verificado de Afromama muestra en formato de lista los fonogramas editados, los cuales pertenecen a músicos que integran su staff y que participan asiduamente de las performances en vivo.

Cada fonograma posee una imagen de portada que contiene un mismo logotipo de identificación. Los rasgos estilísticos de la imagen varían sutilmente según cada portada. Los colores del fondo cambian de tonalidades entre un bordó oscuro y morado, la inscripción debajo del logo permite leer el nombre del fonograma y, por debajo, el nombre de la JBM junto al del autor de la obra.

La uniformidad de rasgos estéticos en las portadas, junto con la visualización de las obras en formato lista, generan un efecto de homogeneización entre los fonogramas, dotándolos de un sentido de identificación con el perfil del usuario que los contiene, que, a su vez, incrementa el potencial de circulación que obtienen por sí solos en tanto los conecta con los seguidores de la JBM (ver figura 9).
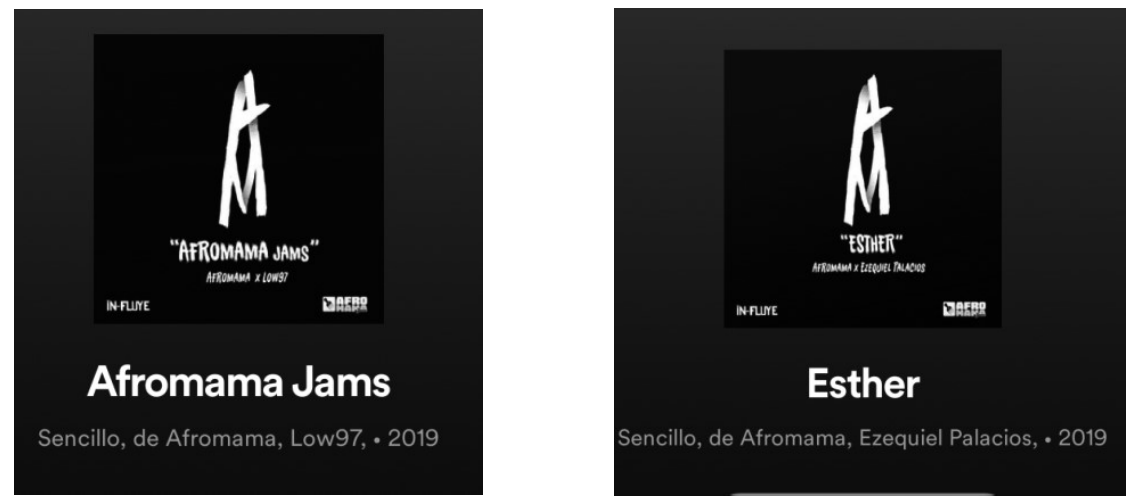

Figura 9. Visualización de los fonogramas en formato individual. Los cuadrados superiores corresponden a las imágenes de portada de cada uno de ellos (Fuente: perfil de usuario de Afromama en Spotify).

El proceso de identificación es similar al que generan los sellos discográficos -majors e independientes- cuando incluyen artistas en sus catálogos. Los sellos, además de avocarse a la edición de fonogramas, también suelen producir música en vivo como mecanismo de exhibición de su catálogo junto con diferentes tareas de circulación (Albornoz y Gallego, 2012; Boix, 2015, 2016, 2017).

\section{Relaciones online y offline}

Para describir las particularidades registradas en las relaciones entre ambas instancias, se tuvieron en cuenta, por un lado, seis tipos de relaciones que aparecen en los sistemas de intercambios descriptos por José Luis Fernández (2020), las cuales son 
Emiliano Vargas

Jams de Black Music en Buenos Aires: relaciones entre performances en vivo y sus mediatizaciones de Spotify

susceptibles de ser aplicadas tanto a las relaciones entre las mediatizaciones y sus materialidades como a los intercambios cara a cara, es decir, abarca las relaciones entre las instancias online y offline. Se trata de relaciones que sirven para "precisar características entre los diferentes modos de contacto, construcciones de grupos y conflictos entre ellos" (Fernández, 2020, p. 27). Las relaciones son: reciprocidad, simetría, complementariedad, presuposición, enunciación e interacción.

Por otro lado, habiendo quedado expuestas las relaciones y características de lo que se puede considerar performance grabada y performance en vivo, se utilizaron las seis cualidades descriptas por Alejandro Frigerio (1992), las cuales rescatan "reglas, estructuras, principios o valores" (p. 3) que distinguen a la performance artística afroamericana. El interés en las cualidades elaboradas por Frigerio reside en que fueron creadas a principios de la década del 90, es decir, la tipología pertenece a un momento mediático previo $^{25} \mathrm{y}$, por lo tanto, su implementación para un análisis desde el momento mediático actual abre nuevos interrogantes de interés en torno a la relevancia de las nuevas mediatizaciones para el sostenimiento de las reglas, estructuras y principios que menciona el autor en fenómenos contemporáneos como las JBM. Las cualidades son: multidimensional, participativa, ubicuidad en la vida cotidiana, conversacional, estilo individual y función social.

Con respecto a la tipología expuesta por Fernández, se ha registrado que las relaciones establecidas entre los sistemas de intercambio discursivo de las JBM tienden a las del tipo reciprocidad, simetría, complementariedad e interacción.

Reciprocidad y simetría aparecen cuando, a través de los intercambios, se parte de relaciones asimétricas, pero se tiende a la simetría a un mediano o largo plazo. Un claro ejemplo fue descripto en los momentos en que la instancia online cubre falencias de la performance en vivo o viceversa. Por ejemplo: la ausencia de clásicos en la playlist de Skill Session es suplida con la presencia de estos durante el vivo. Incluso, entre relaciones que se pueden establecer entre una JBM y otra, la simetría aparece en los puntos de encuentro entre ambas, tanto en términos de uso de dispositivos técnicos (playlists), géneros y estilos dentro de un tipo de black music construido a partir de una herencia afronorteamericana en detrimento de géneros considerados afroargentinos -como un tipo de candombe o el pericón-, como también en prácticas sociales, como un tipo de performance en vivo que pondera el reconocimiento de la pieza ejecutada por sobre rasgos ex tempore.

Las relaciones de complementariedad aparecen cuando los patrones son fundamentalmente diferentes y llevan a intercambios del tipo de la sumisión o del aprendizaje, cubriendo necesidades que suelen ser más explicitas; por ejemplo, al realizar

${ }_{25}$ Momento caracterizado por un sistema de intercambio discursivo entre un emisor y muchos receptores, también denominado modelo estrella, que, en las vidas de lo musical, garantizó el momento de auge de la industria discográfica. 
intercambios a través de la edición de fonogramas de los músicos que integran el staff de Afromama, los cuales permiten incrementar el nivel de circulación de sus obras. A su vez, los músicos ejecutan sus composiciones en la instancia offline, contribuyendo a su sostenimiento.

Por su parte, las relaciones de interacción son consideradas las más evidentes y están vinculadas al tipo de fenómeno que se estudia. Los distintos tipos de interacción van desde las establecidas entre músicos y públicos y músicos entre sí en la instancia offline, registradas, por ejemplo, en la necesidad de reconocimiento de la pieza que se ejecuta. Y a nivel online, solo por citar un ejemplo de los que se abordaron, se puede nombrar a las variadas posibilidades interactivas que ofrecen las playlist.

Se destaca que los mismos tipos de relaciones fueron registrados también como predominantes mediante operaciones de transposición en un trabajo anterior enfocado en las mediatizaciones de Instagram, lo cual evidencia el tipo de relaciones que pondera el conjunto del ecosistema mediático de las JBM (Vargas, 2020, p. 91).

Por último, se exhiben las seis cualidades elaboradas por Alejandro Frigerio. Resulta importante subrayar que son reconocibles en su totalidad solo al analizar ambas instancias (offline y online).

(i) Multidimensional. Se trata de una cualidad que se logra mediante la "superposición de géneros diferentes" (Frigerio, 1992, p. 3). Se registra en la conjugación de géneros musicales y prácticas que pertenecen a distintas tradiciones musicales comprendidas dentro de la semiosfera en las instancias offline y online. Ofrece la ejecución de versiones, en ocasiones fusionadas con freestyle, y la participación del DJ como parte de una misma propuesta.

(ii) Participativa. Es una cualidad que se registra en tanto "no existe una distinción tan rígida como en la cultura occidental entre los performers y el público" (Frigerio, 1992, p. 5). Aparece en tres rasgos: para el caso A, en el rol activo del público a través de la necesidad del reconocimiento de las piezas ejecutadas; para el caso $\mathrm{B}$, a través de un mayor nivel de participación en el que el público se vuelve performer en una misma velada o en veladas sucesivas; $y$, en tercer lugar, a partir de las posibilidades de interacción que posibilita la plataforma (compartir, megustear, agregar fonogramas a otras playlists).

(iii) Ubicuidad en la vida cotidiana. Lograda exclusivamente a partir de las mediatizaciones en Spotify en tanto les permiten a públicos y usuarios/oyentes interactuar cotidianamente y en movilidad con las versiones de base encapsuladas en los fonogramas.

(iv) Conversacional. A nivel de ejecución, las conversaciones entre coros e instrumentos se reducen a lapsos de improvisación y arreglos. En el vínculo perfor- 
mer-público se registra a partir de la necesidad de reconocimiento de la pieza musical. A nivel online, desborda gracias a las posibilidades de interacción y ubicuidad.

(v) Estilo individual. Registrado en lapsos que se liberan a la improvisación y al ejecutar las versiones originales mediante su/s autor/es. A nivel online, con los fonogramas pertenecientes a artistas que se suben a escena en el vivo.

(vi) Función social. Registrado principalmente a partir de las relaciones de reciprocidad, simetría y complementariedad.

\section{Conclusiones provisorias y nuevos interrogantes}

El tipo de performance construido por las JBM se basa en réplica o cover, covering y canción paralela. La improvisación ocupa un lugar secundario desde el que se elaboran distanciamientos entre los ideales de encapsulación y participación. Ambas propuestas poseen espacios físicos en los que las performances se llevan a cabo con mayor regularidad sin perder sus atributos itinerantes.

Las mediatizaciones en Spotify muestran que las piezas ejecutadas se encuentran encapsuladas dentro de las playlists en sus versiones originales o de base.

A través de Spotify, generan perfiles sonoros y se garantiza una parte de la circulación de los fonogramas de artistas de bajo reconocimiento.

El uploading musical de Spotify permite incrementar la autonomía productiva de Afromama, JBM que en 2019 comenzó a producir patrimonio musical encapsulado, ocupándose de la producción y edición de fonogramas de artistas que forman parte de su staff, además de la circulación y ubicuidad de los productos para su consumo.

Las plataformas analizadas absorben tareas que en otros momentos mediáticos pertenecían exclusivamente a artistas, técnicos, sellos y medios masivos. Por ejemplo, a nivel de producción, la masterización y el diseño de imágenes de portadas, $\mathrm{o}$ a nivel de circulación, mediante acciones de interacción discursiva. Se profundizará sobre estos aspectos en futuros trabajos.

A través del perfil de artista verificado, se genera un efecto de homogeneización entre los fonogramas editados, a través del cual se gestan identificaciones con el colectivo mayor que los contiene.

La performance en vivo de Afromama reproduce el estilo catálogo de su playlist apelando a las versiones y composiciones de los artistas de su staff. En ocasiones, se invita a artistas nacionales consagrados que también se encuentran encapsuladas en su playlist para que ejecuten piezas musicales.

La performance en vivo de Skill Session contiene aspectos de los estilos -catálogo y emergente- descriptos en el apartado sobre playlists. Emergente porque se trata de una propuesta más inclusiva en la que no existe un staff cerrado, pero, a nivel de ejecución, 
Austral Comunicación

Volumen 9, número 2 (Diciembre de 2020): 299-323. ISSN 2313-9129

sus rasgos se asemejan al estilo catálogo en tanto hay un predominio de clásicos de la semiosfera que no están encapsulados en su playlist.

Las relaciones establecidas entre las instancias offline y online tienden a la reciprocidad, simetría, complementariedad e interacción. Las cualidades de la performance artística afroamericana aparecen en su totalidad solo al analizar la tríada composición-performance-improvisación a nivel online y offline como parte de un todo.

Los resultados arrojan dos nuevas hipótesis: (i) en el momento mediático actual, el tipo de performance musical de las JBM debe necesariamente ser comprendido a través de un análisis holístico de sus vidas offline/online que abarque al conjunto del ecosistema mediático y no solo la distinción elaborada por distintos autores entre performance en directo y performance grabada; y (ii) el incremento de la autonomía productiva de las JBM deriva en un crecimiento del conjunto del patrimonio cultural que producen.

Es decir, quedan abiertos varios interrogantes en relación con las formas de producción musical que predominarán en estos colectivos hacia el futuro. ¿Se incrementará el volumen de producciones fonográficas y los perfiles de artista verificado en las JBM? ¿Habrá una equiparación en la densidad de producciones online y offline? O, por el contrario, ¿se trata de una excepción y no de un proceso de transformación más amplio? Hay un camino fértil para el aumento de la producción del patrimonio musical encapsulado, pero todavía se trata de un proceso incipiente.

\section{Referencias}

Afromama, Música Negra en el Corazón de Palermo (2 de noviembre de 2018). Alerta Cultural. Obtenido de http://www.alertacultural.com/ Afromamas-musica-negra-en-el-corazon-de-palermo/.

Albornoz, L. A. y Gallego, J. I. (2012). La industria de la música popular en España: los sellos independientes en la era digital. E-Compós, 15(2).

Benson, B. E. (2003) Between Composition and Performance. En The improvisation of musical dialogue: A phenomenology of music (pp.16-32). Cambridge University Press.

Boix, O. A. (2015). Amigos sí, jipis no: cómo ser un "profesional" de la música en un "sello" de la ciudad de La Plata. Revista Ensambles, (2).

Boix, O. A. (2016). Música y profesión: organizaciones socio musicales y trayectorias emergentes en la ciudad de La Plata (2009-2015) (Tesis Doctoral). Facultad de Humanidades y Ciencias de la Educación, Universidad Nacional de La Plata.

Boix, O.A. (2017). Estado y organizaciones musicales en las configuraciones emergentes en los años 2000 en Argentina. Resonancias, 21(40), 129-144.

Cole, M. (2013). Black Music \& Identity: How Commodification Changed The Narratives of $R \& B$ Music. Obtenido de https://www.academia.edu/11223984/Black_Music_and_ Identity_How_Commodification_Changed_The_Narratives_of_R_and_B_Music. 
Emiliano Vargas

Jams de Black Music en Buenos Aires: relaciones entre performances en vivo y sus mediatizaciones de Spotify

Cook, N. (2013). Introduction. En Beyond the score: Music as a Performance (p. 6). Nueva York: Oxford University Press.

Corti, B. (2015). Jazz Argentino. La música "negra" del país "blanco". Gourmet Musical Ediciones, Buenos Aires.

Fernández, J. L. (Coord.). (2014). Postbroadcasting: Innovación en la industria musical. Buenos Aires: La Crujía.

Fernández, J. L. (2017). Plataformas Mediáticas. Elementos de análisis y diseño de nuevas perspectivas. Buenos Aires: La Crujía.

Fernández, J. L. (2020). Mediatizaciones y unidades de análisis: aproximaciones a la complejidad. En Busso, M. P. y Gindin, I. L. (2020). Zonas de la mediatización: propuestas para el estudio de plataformas, redes e interfaces (pp. 11-41). Rosario: UNR Editora.

Frigerio, A. (1992). Artes negras: una perspectiva afrocéntrica. Revista de Estudios Afro-Asiáticos, (23), 175-90.

Frigerio, A. y Lamborghini, E. (2011). Los afroargentinos: formas de comunalización, creación de identidades colectivas y resistencia cultural y política. Aportes para el desarrollo humano en Argentina, (5), 5-51.

Greimas, A. J. (1968). Le condizioni per una semántica scientifica. En Semantica strutturale (pp. 13-14). Milán: Rizzoli Editori.

Jáuregui, J. (2015). Streaming musical en Spotify: ubicuidad entre géneros y estados de ánimo. Inmediaciones de la Comunicación, (10), 76-90.

Jociles Rubio, M. I. (2018). La observación participante en el estudio etnográfico de las prácticas sociales. Revista Colombiana de Antropología, 54(1), 121-150.

Kawulich, B. B. (2005). La observación participante como método de recolección de datos. Forum Qualitative Social Research, 6(2), art. 43.

Latham, A. (2017). Diccionario enciclopédico de la música. México D. F.: Fondo de Cultura económica,

Lenarduzzi, V. (2012). Una Estética Relacional. En Placeres en Movimiento. Cuerpo Música y Baile en la "Escena Electrónica" (pp. 79-1). Buenos Aires: Aidos.

López Cano, R. (2018). Música Dispersa: Apropiación, influencias, robos y remix en la era de la escucha digital. Barcelona: Musikeon Books.

Lotman, I. (1998a). El Texto y la Función. En La semiosfera II Semiótica de la cultura, del texto, de la conducta y del espacio (pp. 116-124, Trad. Desiderio Navarro). Madrid: Cátedra/Gedisa.

Lotman, I. (1998b). Sobre el Mecanismo Semiótico de la Cultura. En La semiosfera III Semiótica de las artes y de la cultura (3a ed., pp. 168-213, Trad. Desiderio Navarro). Valencia: Universitat de València.

Marino, G. (2020). Frammenti di un disco incantato. Teorie semiotiche, testualità e 
Austral Gmunicación

Volumen 9, número 2 (Diciembre de 2020): 299-323. ISSN 2313-9129

generi musicali. Turín: I Saggi di Lexia, Università degli Studi di Torino, Gioacchino Onnorati editore.

Mendizábal, N. (2006). Los componentes del diseño flexible en la investigación cualitativa. Estrategias de investigación cualitativa, (1), 65-106.

Morgan, B. A. (2020). Revenue, access, and engagement via the in-house curated Spotify playlist in Australia. Popular Communication, 18(1), 32-47.

Neal, M. A. (1999). What the Music Said: Black Popular Culture and Black Public Culture. Nueva York/Londres: Routledge.

Nettl, B. y Russell, M. (eds.). (2004). Introducción. Un Arte Relegado por los Eruditos. En En el transcurso de la interpretación ( $\left.{ }^{\mathrm{a}} \mathrm{ed} ., \mathrm{pp} .10-11\right)$. Madrid: Ediciones AKAL.

Ripani, R. J. (2006). The New Blue Music. Changes in the Rhythm \& Blues, 1950-1999. Wm. B. Eerdmans Publishing.

Sampieri, R. y Collaro, C. (2006). Muestras de orientación hacia la investigación cualitativa. En Metodología de la investigación (4 ed., pp. 567-571). México: McGrow Hill.

Spotify Eliminará un Servicio Para artistas Independientes (1 de julio de 2019). Infobae. Obtenido de https://www.infobae.com/america/tecno/2019/07/01/ spotify-eliminara-un-servicio-para-artistas-independientes/.

Steimberg, O. (1993). Proposiciones sobre el género. En Semiótica de los medios masivos. El pasaje a los medios de los géneros populares (pp. 47-84). Buenos Aires: Atuel.

Tagg, P. (1989). Open letter: 'Black music', 'Afro-American music' and 'European music'. Popular Music, 8(3), 285-298.

Tarasti, E. (2002). On the semiosis of musical improvisation: From Mastersingers to Bororo indians. En Signs of music: a guide to musical semiotics (pp. 179-196). Nueva York: Mouton de Gruyter.

Vargas, E. (2020). Mediatizaciones en Jams de Música de Raíz Afronorteamericana en Buenos Aires. En Busso, En Busso, M. P. \& Gindin, I. L. Zonas de la mediatización: propuestas para el estudio de plataformas, redes e interfaces (pp. 61-94). Rosario: UNR Editora.

Vega, N. R. (2020). El ascenso del freestyle de competencia en Chile: la batalla de gallos como forma renovada de hacer y consumir el hip-hop. Contrapulso-Revista Latinoamericanad de Estudios en Música Popular, 2(2), 65-79.

Vuelve la fiesta Afromama a Rosario (21 de mayo de 2017). Conclusión. Obtenido de https://www.conclusion.com.ar/espectaculos/ vuelve-la-fiesta-afromama-a-rosario/05/2017/.

Webster, J. (2019). Music on-demand: A commentary on the changing relationship between music taste, consumption and class in the streaming age. Big Data \& Society, 6(2). 